\title{
N.I. KASHIRINA
}

V.E. Lashkaryov Institute of Semiconductor Physics, Nat. Acad. of Sci. of Ukraine (41, Nauky Ave., Kyiv 03028, Ukraine; e-mail: n_kashirina@mail.ru)

\section{APPLICATION OF QUANTUM FIELD THEORY METHODS TO THE DEVELOPMENT OF THE TRANSLATIONAL-INVARIANT \\ PACS 71.38, 71.38.+i, 73.20.D, 74.80.D POLARON AND BIPOLARON THEORY}

\begin{abstract}
The polaron and bipolaron energy functionals obtained in the framework of quantum field theory have been studied. Exact analytical expressions for the effective functionals are derived in terms of the two-parametrical trial function for a polaron and the three-parametrical one for a bipolaron. Variational solutions are found for the energies of the systems under study in the case of the intermediate values of Fröhlich electron-phonon coupling constant, $4 \leq \alpha \leq 20$. Keywords: polaron, bipolaron, Fröhlich Hamiltonian, electron-phonon interaction, hightemperature superconductivity.
\end{abstract}

\section{Introduction}

Besides the known applications associated with the attempts to construct the theory of high-temperature superconductivity on the basis of the bipolaronic mechanism $[1,2]$, the interest in the idea of polaron is related to the fact that the model problems, in which various aspects of the polaron theory are considered and which have a simple formulation, are very convenient for the development of theoretical physics methods aimed at studying the interaction between charged particles and quantum fields. The polaron and bipolaron states, as well as numerous applications of the polaron theory to various domains of condensed matter physics, are used in a huge number of works, reviews, and monographs (see, e.g., work [3] and references therein).

In work [4] with the use of the quantum field theory methods, a consistent polaron theory making allowance for the translational invariance was developed. The results obtained in the strong-coupling limit reproduce those of Pekar's polaron theory [5]. In addition, the results obtained by A.V. Tulub in work [4] in the weak-coupling limit and in the intermediate (closer to the weak limit) coupling range turned out close to Feynman's ones obtained in the framework of the path-integration method. In work [6], it was found that, at large values of Fröhlich electronphonon coupling constant $\alpha$, the method proposed by A.V. Tulub brings about the lowest polaron ener-

(C) N.I. KASHIRINA, 2014

1088 gies in comparison with other methods. The binding energy was calculated using approximate expressions obtained, strictly speaking, in the limit $\alpha \rightarrow \infty$.

Of great interest would be the derivation of exact mathematical expressions for the effective polaron and bipolaron functionals and the given set of trial functions in the framework of field theory. For this purpose, it is necessary to obtain, without any approximations, maximally simplified analytical expressions for the polaron and bipolaron energy functionals that would allow one to carry out the procedure of numerical minimization in the range of the values of electron-phonon coupling parameter $4 \leqslant \alpha \leqslant 20$, which is the most relevant for the bipolaron theory.

\section{Tulub's Polaron and Bipolaron Functionals}

The procedure to derive the polaron [4] and bipolaron $[7,8]$ functionals taking the recoil effects into account was proposed in work [4]. It includes the following steps. 1) Two classical canonical Lee-LowPines transformations [9] are sequentially applied to the Fröhlich Hamiltonian describing the electronphonon interaction. The first transformation excludes the electron coordinate, and the second one corresponds to the shift of the phonon creation, $\mathbf{a}_{\mathbf{k}}^{+}$, and annihilation, $\mathbf{a}_{\mathbf{k}}$, operators. 2) In the obtained Hamiltonian, the following terms are left:

$H_{0}=\sum_{\mathbf{k}} \omega_{\mathbf{k}}^{0} \mathbf{a}_{\mathbf{k}}^{+} \mathbf{a}_{\mathbf{k}}+\frac{1}{2 m^{*}}\left(\sum_{\mathbf{k}} \mathbf{k} f_{\mathbf{k}}\left(\mathbf{a}_{\mathbf{k}}^{+}+\mathbf{a}_{\mathbf{k}}\right)\right)^{2}$.

ISSN 2071-0194. Ukr. J. Phys. 2014. Vol. 59, No. 11 
Here, $m^{*}$ is the effective electron mass, the variational function $f_{\mathbf{k}}$ determines a shift of the phonon operators $\left(\mathbf{a}_{\mathbf{k}} \rightarrow \mathbf{a}_{\mathbf{k}}+f_{\mathbf{k}}\right), \mathbf{k}$ is the wave vector of phonons, and $\omega_{\mathbf{k}}^{0}$ is the frequency of longitudinal optical phonons. In what follows, the dispersion of $\omega_{\mathbf{k}}^{0}$ is neglected.

Expression (1) can be transformed to a diagonal form using the methods of quantum field theory. The determination of the energy is reduced to the averaging of the polaron functional over the eigenfunctions of operator (1) and the following minimization over $f_{\mathbf{k}}$. In the strong-coupling limit, a simplified variation procedure is used: the functional is varied over the parameters of the function $f_{\mathbf{k}}$, assuming the form of the latter to be preliminary fixed, rather than over the function $f_{\mathbf{k}}$ itself.

The analytical expression for Tulub's polaron functional obtained in work [4] for the zero total momentum of a polaron and the trial function $f_{\mathbf{k}}$ looks like

$E_{\mathrm{PT}}=\Delta E+2 \sum_{\mathbf{k}} V_{k} f_{k}+\sum_{\mathbf{k}} f_{k}^{2}$.

Here, $\Delta E$ is the eigenvalue of Hamiltonian (1) (the Feynman units $2 m^{*}=1, \hbar=1$, and $\omega_{k}^{0}=1$ are used, so that the energy unit is $\hbar \omega_{k}^{0}$ and the length unit is $\left.L_{0}=\sqrt{\hbar / 2 m \omega_{k}^{0}}\right), V_{k}=k^{-1} \hbar \omega_{k}^{0} \sqrt{4 \pi \alpha L_{0} / V}$, $\alpha=\frac{e^{2}}{2 \hbar \omega_{k}^{0} L_{0}}\left(\frac{1}{\epsilon_{\infty}}--\frac{1}{\epsilon_{0}}\right)$, where $V$ - the volume of the crystal, $\epsilon_{\infty}$ and $\epsilon_{0}$ are the high-frequency and static dielectric permittivities, respectively.

The bipolaron functional can be found after changing in the bipolaron Hamiltonian to the coordinates of the center of mass, similarly to what was done in work [10]. Afterward, the calculation procedure described above when obtaining functional (2) is repeated. The three-parametrical bipolaron functional obtained using Tulub's method in the framework of quantum field theory was analyzed in work [8]. It can be written in the form

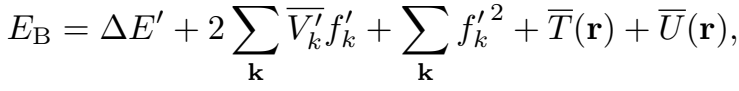

where $\overline{V_{k}^{\prime}}=2 V_{k}\langle\Psi|\cos \mathbf{k r} / 2| \Psi\rangle ; \bar{T}$ and $\bar{U}$ are the average values of the kinetic energy and the energy of electron repulsion in the coordinates of the center of mass:; $\mathbf{r}=\mathbf{r}_{1}-\mathbf{r}_{2} ; \mathbf{R}=\left(\mathbf{r}_{1}+\mathbf{r}_{2}\right) / 2 ;$ and $\mathbf{r}_{1}$ and $\mathbf{r}_{2}$ are the radius vectors of electrons 1 and 2 , respectively. The coordinate $\mathbf{R}$ of the center of mass is excluded from Eq. (3) using the first canonical LeeLow-Pines transformation [9]. The primes are introduced to distinguish the quantities $\Delta E$ and $\Delta E^{\prime}$ and the functions $f_{k}$ and $f_{k}^{\prime}$ entering Eqs. (2) and (3), respectively.

The two-parametrical trial polaron function is selected in the form

$f_{k}=-N V_{k} \exp \left(-k^{2} / 2 a^{2}\right)$,

where $N$ and $a$ are variational parameters. The bipolaron functional (3) is minimized with a trial variational function, whose phonon part looks like

$f_{k}^{\prime}=-N \overline{V_{k}^{\prime}} \exp \left(-k^{2} / 2 \mu\right)$,

and the coordinate part like

$\Psi(\mathbf{r})=\left(2 / \pi b^{2}\right)^{3 / 4} \exp \left(-r^{2} / b^{2}\right)$,

where $N, \mu$, and $b$ are variational parameters.

In the strong-coupling limit, the first term in Eq. (2) plays the role of the kinetic energy of an electron in the phonon field. In the general case of intermediate electron-phonon coupling constant values, the terms corresponding to the kinetic energy of electrons stand out and do not coincide with the values of $\Delta E$ for a polaron and $\Delta E^{\prime}+\bar{T}$ for a bipolaron.

Putting the integration limits over the phonon wave vector equal to infinity, we obtain

$$
\begin{aligned}
& \Delta E=\frac{1}{2 \pi^{2}} \int_{0}^{\infty} d k k^{4} f_{k}^{2} \omega_{k} F\left(\omega_{k}\right), \\
& F\left(\omega_{k}\right)=\frac{1}{2 \pi i} \int_{C} \frac{\sqrt{s}}{\left(s-\omega_{k}^{2}\right)^{2}} \frac{1}{D(s)} d s, \\
& D(s)=1-\frac{2}{3(2 \pi)^{3}} \int \frac{k^{2} f_{k}^{2} \omega_{k}}{s-\omega_{k}^{2}} d^{3} \mathbf{k},
\end{aligned}
$$

where $\omega_{k}=1+k^{2} / 2$. The choice of the integration path $C$ in the plane of the complex variable $s$ was discussed in work [4].

The quantity $\Delta E^{\prime}$ in the bipolaron functional (3) can be obtained from $\Delta E$ by substituting $f_{k}^{\prime}$ for $f_{k}$.

Expression (6) can be simplified very much. A lot of integrals can be calculated analytically. The integrals with poles on the real axis are calculated in the principal value sense.

In the case of the variational polaron function $f_{k}$ and when integrating within infinite limits, the real and imaginary parts of expression (7) look like

$\operatorname{Re} D\left(\omega_{k}^{2}\right)=1+\lambda \nu(y), \quad \operatorname{Im} D\left(\omega_{k}^{2}\right)=k^{3} f_{k}^{2} / 6 \pi$, 


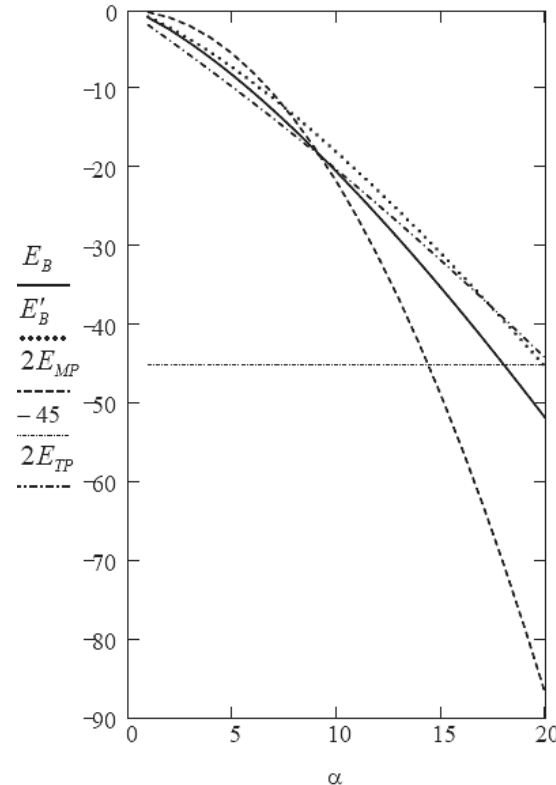

Dependences of the energies of a bipolaron, $E_{\mathrm{B}}$, doubled Miyake strong-coupling polaron [15], $2 E_{\mathrm{MT}}$, and doubled Tulub polaron, $2 E_{\mathrm{TP}}$, on the Fröhlich coupling constant at $\eta=$ $=\varepsilon_{\infty} / \varepsilon_{0}=0$, and the corresponding dependence of the bipolaron energy $E_{\mathrm{B}}^{\prime}$ calculated at $\eta=0.1$. The horizontal dashdotted line marks an energy of -45 and was drawn to make the comparison of the results obtained in this work with those of work [12] convenient. The energy $E_{\mathrm{TP}}$ was calculated using the two-parametrical function $f_{\mathbf{k}}$, and the energies $E_{\mathrm{B}}$ and $E_{\mathrm{B}}^{\prime}$ using the three-parametrical function $f_{\mathbf{k}}^{\prime}$ and expressions (9)-(13) for a polaron and the corresponding expressions for a bipolaron

Dependences of the bipolaron energy on the electron-phonon coupling parameters: current calculations $\left(E_{\mathrm{B}}\right)$, extrapolation of the strong-coupling limit $1 / \lambda=0\left(E_{\mathrm{Bs}}\right)$, and the energy calculated in work $[13]\left(E_{\mathrm{BK}}\right) . \eta=\varepsilon_{\infty} / \varepsilon_{0}$

\begin{tabular}{|l|l|l|l|}
\hline \multicolumn{3}{|c|}{$\alpha=9$} \\
\hline$\eta$ & $-E_{\mathrm{B}}$ & $-E_{\mathrm{Bs}}$ & $-E_{\mathrm{BK}}$ \\
\hline 0 & 19.08 & 35.69 & 24.93 \\
0.01 & 18.86 & 35.25 & 24.65 \\
0.1 & 16.79 & 31.00 & 22.07 \\
\hline \multicolumn{3}{|c|}{$\alpha=7$} & $-E_{\mathrm{BK}}$ \\
\hline$\eta$ & $-E_{\mathrm{B}}$ & $-E_{\mathrm{Bs}}$ & 16.23 \\
\hline 0 & 13.02 & 21.60 & 16.05 \\
0.01 & 12.24 & 21.32 & 14.60 \\
\hline 0.1 & 10.89 & 18.75 & \\
\hline
\end{tabular}

where

$$
\begin{aligned}
& \nu(y)=1-y e^{-y^{2}} \int_{0}^{y} e^{t^{2}} d t-\xi(y) e^{-\xi(y)^{2}} \int_{\xi(y)}^{\infty} e^{-t^{2}} d t, \\
& \xi(y)=\sqrt{y^{2}+4 / a^{2}}, y=k / a, \lambda=4 N^{2} \alpha a / 3 \sqrt{2 \pi} .
\end{aligned}
$$

It is worth paying attention to the fact that the account for the phonon spectrum confinement by a threshold value of the wave vector results in more cumbersome expressions. Not only does the analytical expression for the function $D(s)$ change, but also the form of polaron and bipolaron functionals. Namely, there appears an additional term, the general form of which is given in work [11]. In this work, the limits of integration over the wave vector are not confined.

The exact formulas obtained for quantity (6) with the use of the trial variational function $f_{k}$ (Eq. (4)) and provided infinite integration limits are as follows:

$\Delta E(a, N, \alpha)=\Delta E_{0}(a, N, \alpha)+\Delta E_{1}(a, N, \alpha)$,

$\Delta E_{0}=\frac{3 a^{2}}{16} q_{0}(a, N, \alpha)$,

$\Delta E_{1}=\frac{3 a^{2}}{16} q_{1}(a, N, \alpha)$,

$q_{0}=\frac{1}{1+\frac{1}{\lambda}-\frac{\sqrt{\pi}}{a} \exp \left(4 / a^{2}\right)(\operatorname{erf}(2 / a)-1)}$,

$q_{1}=\frac{2}{\sqrt{\pi}} \int_{0}^{\infty} d y \frac{e^{-y^{2}}(1-\tilde{\Omega}(y)) \widetilde{\eta}(y)}{(\nu(y)+1 / \lambda)^{2}+\pi y^{2} \exp \left(-2 y^{2}\right) / 4}$.

Here, $\widetilde{\eta}(y)=\left(y^{2}+2 / a^{2}\right) /\left(y^{2}+4 / a^{2}\right)$,

$$
\begin{aligned}
& \tilde{\Omega}(y)=2 y^{2}\left[\left(1+2 \xi(y)^{2}\right) \xi(y) e^{\xi(y)^{2}} \times\right. \\
& \left.\times \int_{\xi(y)}^{\infty} e^{-t^{2}} d t-\xi(y)^{2}\right] .
\end{aligned}
$$

In the limit $a \gg 1$ and $\alpha \gg 1$, we obtain $\Delta E_{0} \approx 3 a^{2} / 16, \quad \xi(y) \approx y, \quad \widetilde{\eta}(y) \approx 1$.

Let us introduce the new notation for this limit: $q_{1} \approx q(1 / \lambda), \quad \tilde{\Omega}(y) \approx \Omega(y)$.

ISSN 2071-0194. Ukr. J. Phys. 2014. Vol. 59, No. 11 
Hence, in the strong-coupling limit, expressions (12) and (13) can be approximately written as follows:

$$
\begin{aligned}
& q(1 / \lambda)=\frac{2}{\sqrt{\pi}} \int_{0}^{\infty} d y \times \\
& \times \frac{e^{-y^{2}}(1-\Omega(y))}{(\nu(y)+1 / \lambda)^{2}+\pi y^{2} \exp \left(-2 y^{2}\right) / 4}, \\
& \Omega(y)=2 y^{2}\left[\left(1+2 y^{2}\right) y e^{y^{2}} \int_{y}^{\infty} e^{-t^{2}} d t-y^{2}\right] .
\end{aligned}
$$

The approximate expressions (14) (at $N=1$ ) and (15) coincide with the quantities $q(1 / \lambda)$ and $\Omega(y)$ of work [4].

At large values of electron-phonon coupling constant, there emerges a delta-like peculiarity in the integrands of expressions (12) and (14). It appears because the quantity $\operatorname{Re} D\left(\omega_{k}^{2}\right)$ defined by formula (8) passes through zero and becomes negative. This peculiarity does not appear at the small enough values of Fröhlich coupling constant, $\alpha \leq 10$. At the same time, at $10 \leq \alpha \leq 20$, the integrals in expression (6) can be calculated numerically with a good accuracy.

In the variational bipolaron function $f_{k}^{\prime}$, we took the additional variational parameter $N$ into account, the role of which was discussed in work [6]. Therefore, the values of bipolaron energy in the strong-coupling limit at $\alpha \rightarrow \infty$ for $\eta=0$, which were obtained using the exact expressions $(9)-(13)$ - namely, $E_{\mathrm{Bs}} \approx$ $\approx-0.440959 \alpha^{2}$, with the principal value of integral (12) being calculated numerically - turned out a little smaller than the value obtained in work [7] for $N=2$ (without varying the bipolaron functional over this parameter). The results obtained in the strong-coupling approximation are valid with a good accuracy in the interval $\alpha \geq 35$. The value $N=\sqrt{2}$ coincides with the optimum value for Tulub's strong-coupling polaron functional [6] in the limit $1 / \lambda \rightarrow 0$. In work [8], the bipolaron functional was optimized with respect to the parameter $N$. However, the calculations were carried out for the approximate value $\Delta E$ given in work [4]. In addition, the approximation $1 / \lambda \approx 0$ was used, which made the bipolaron functional considerably simpler, but resulted in the underestimated values of bipolaron energy in the interval of the relatively small values of electron-phonon coupling parameter, $\alpha \leq 20$. At the same time, the application of the exact formulas (9)-(13) for a polaron and the corresponding expressions for a bipolaron with the additional variational parameter $N$ in the interval $4 \leq \alpha \leq 20$ brought about lower bipolaron energies in comparison with those in work [12]. The authors of that work used the approximate strong-coupling formulas given in work [4] for the positive increment $\Delta E$, but abandoned the approximation $1 / \lambda=0$ in expression (14).

\section{Calculation Results}

The polaron energy was calculated, by using the exact formulas (9)-(13) for the term $\Delta E$ in functional (2). The quantity $\Delta E^{\prime}$ in Eq. (3) can be obtained from $\Delta E$ by changing the notation of variational parameters. The analytical expressions for other terms in bipolaron functional (3) can be found in work [8].

In Table, the results obtained for the bipolaron energy $E_{\mathrm{B}}$ using the exact formulas for the term $\Delta E^{\prime}$ are shown, as well as the corresponding values obtained in works [8,13], for $\alpha=9$ and 7 , and various values of the parameter $\eta=\varepsilon_{\infty} / \varepsilon_{0}$. The quantity $E_{\mathrm{Bs}}$ corresponds to the extrapolation of the approximation $1 / \lambda=0$ to the region of the relatively small values of parameter $\alpha$ (this extrapolation was done in works $[7,8]$ ), and $E_{\mathrm{BK}}$ is the bipolaron energy obtained in work [13] using the Buimistrov-Pekar method [14]. Note that the values of $E_{\mathrm{B}}$ and $E_{\mathrm{BK}}$ were obtained in the framework of the variational technique without any approximations in the initial functionals. Therefore, they can be regarded as the upper limits of the bipolaron energy. At the same time, the $E_{\mathrm{Bs}}$-values were obtained by varying the approximate functional in the limit $1 / \lambda=0$.

In Figure, the results of numerical calculations for the polaron and bipolaron energies are shown. For comparison, the corresponding dependence for the doubled energy of a Miyake strong-coupling polaron [15] is plotted. The analysis of the data obtained for the bipolaron energy testifies that the extrapolation of the strong-coupling limit (the approximation $1 / \lambda \approx 0)$ to the region of the examined $\alpha$-values is invalid, because this approximation gives rise to the substantially underestimated values of bipolaron energy. The calculations carried out in the framework of Tulub's method without use of the approximate 
expressions for the polaron and bipolaron functionals demonstrated that the critical value of Fröhlich coupling constant in the limit $\eta \rightarrow 0$, below which the bipolaronic state does not exist, equals $\alpha_{c}=10$. This value does not expand the interval of the bipolaron existence in comparison with other methods. As an example, the lower values of bipolaron energy, which were obtained in work [13] for the same parameter values, are quoted in Table.

At the same time, in the interval $\alpha \leq 8$, which was studied in work [4], the asymptotics $q(1 / \lambda)=q(0)$ and the application of approximate expressions for the polaron functional in the strong-coupling limit did not - expectedly - result in the underestimation of the polaron energy. The author of work [4] saw the reason for why the electron-phonon coupling constants are restricted rather strictly by relatively small values in that the polarons cannot be scattered by optical phonons at all at large coupling constant values, because, as $\alpha$ increases, lattice vibrations with shorter and shorter wavelengths become responsible for the scattering. However, the electronphonon interaction at short wavelengths cannot be described in the framework of the continual approximation, which forms the basis of Tulub's theory. The cut-off of the phonon spectrum by a threshold wave vector also gives rise to a restriction with respect to the constant coupling, beyond which the continual approximation does not work. Analytical calculations of elements in the matrix of the polaron scattering by optical phonons and numerical estimations made for the parameters of some crystals (see work [4]) showed that the maximum values of Fröhlich electron-phonon coupling constant are $\alpha_{\max } \approx 8 \div 9$ for the majority of ionic crystals. Those values reduce, in effect, the applicability region of the strong coupling approximation to zero, because either the weak or intermediate coupling should be realized in real crystals.

The analysis of the dependence of the bipolaron energy on the parameter of the phonon spectrum cutoff is an independent and extremely labor-consuming problem. Therefore, it will be done elsewhere. Here, we would like only to note that, for rather small electron-phonon coupling constants, the positive increment, the analytical form of which is given in work [11], does not lead to substantial variations in the polaron and bipolaron energy magnitudes.
The author expresses her sincere gratitude to A.V. Tulub and V.D. Lakhno for the useful discussion of the results obtained in this work.

1. V.L. Vinetsky, N.I. Kashirina, and E.A. Pashitskii, Ukr. Fiz. Zh. 37, 77 (1992).

2. N.I. Kashirina and V.D. Lakhno, Usp. Fiz. Nauk 80, 449 (2010).

3. N.I. Kashirina and V.D. Lakhno, Mathematical Modeling of Autolocalized States in Condensed Media (Fizmatlit, Moscow, 2013) (in Russian).

4. A.V. Tulub, Zh. Èksp. Teor. Fiz. 41, 1828 (1961).

5. S.I. Pekar, Research in Electron Theory of Crystals (United States Atomic Energy Commission, Washington, DC, 1963).

6. N.I. Kashirina, V.D. Lakhno, and A.V. Tulub, Zh. Èksp. Teor. Fiz. 141, 994 (2012).

7. V.D. Lakhno, Solid State Commun. 152, 621 (2012).

8. V.D. Lakhno, Zh. Èksp. Teor. Fiz. 143, 1033 (2013).

9. T.D. Lee, F.E. Low, and D. Pines, Phys. Rev. 90, 297 (1953).

10. V.L. Vinetskii, O. Meredov, and V.Ya. Yanchuk, Teor. Eksp. Khim. 25, 641 (1989).

11. M Porsch and J. Röseler, Phys. Status Solidi 23, 365 (1967).

12. S.N. Klimin and J.T. Devreese, Solid State Commun. 153, 58 (2013)

13. N.I. Kashirina, V.D. Lakhno, and V.V. Sychov, Phys. Status Solidi B 239, 174 (2003).

14. V.M. Buimistrov and S.I. Pekar, Zh. Èksp. Teor. Fiz. 32, 1193 (1957).

15. S.J. Miyake, J. Phys. Soc. Jpn. 38, 181 (1975).

Received 02.07.14

Translated from Ukrainian by O.I. Voitenko

H.I. Каширіна

ЗАСТОСУВАННЯ МЕТОДІВ

КВАНТОВОЇ ТЕОРІЇ ПОЛЯ ДО РОЗРОБКИ

ТРАНСЛЯЦІЙНО-ІНВАРІАНТНОÏ ТЕОРІЇ

ПОЛЯРОНА ТА БІПОЛЯРОНА

$\mathrm{P}$ е $з$ ю м е

У роботі досліджуються функціонали енергії полярона і біполярона, отримані методом квантової теорії поля. Виведено точні аналітичні вирази для ефективних функціоналів з використанням двохпараметричної пробної функції для полярона і трьохпараметричної для біполярона. Варіаційним методом знайдено значення енергії досліджуваних систем для проміжних значень фрьоліховської константи електрон-фононного зв'язку $4 \leq \alpha \leq 20$. 